\title{
Fauna de besouros bioluminescentes (Coleoptera: Elateroidea: Lampyridae; Phengodidae, Elateridae) nos municípios de Campinas, Sorocaba-Votorantim e Rio Claro-Limeira (SP, Brasil): biodiversidade e influência da urbanização
}

\author{
Vadim Ravara Viviani ${ }^{1,2}$, Mayra Yamazaki Rocha ${ }^{1}$ \& Oskar Hagen ${ }^{1}$ \\ ${ }^{1}$ Laboratório de Bioluminescência e Biotecnologia, Universidade Federal de São Carlos - UFSCar, \\ Campus de Sorocaba, Rod. João Leme dos Santos, Km 110, Itinga, \\ CEP 18052-780, Sorocaba, SP, Brasil \\ ${ }^{2}$ Autor para correspondência: Vadim Ravara Viviani, e-mail: biota.biolum@hotmail.com
}

\begin{abstract}
VIVIANI, V.R., ROCHA, M.Y. \& HAGEN, O. Bioluminescent beetles (Coleoptera: Elateroidea: Lampyridae, Phengodidae, Elateridae) in the municipalities of Campinas, Sorocaba-Votorantim and Rio Claro-Limeira (SP, Brazil): biodiversity and influence of urban sprawl. Biota Neotrop. 10(2): http://www.biotaneotropica. org.br/v10n2/en/abstract?article+bn03010022010.
\end{abstract}

\begin{abstract}
Brazil hosts the richest biodiversity of bioluminescent beetles in the world. However, no surveys on the regional biodiversity have been made. Furthermore, studies on the effect of urbanization on bioluminescent beetle's biodiversity are still missing. Such kind of studies could be especially helpful to select new night environment bioindicators. Bioluminescent species of the Elateroidea superfamily occurring in three large and expanding urban areas in São Paulo State: Campinas, Sorocaba-Votorantim and Rio Claro-Limeira municipalities, were catalogued. In Campinas, the occurrence of bioluminescent beetles in different sites has been monitored during the past 20 years. There were 26 species in Campinas, 21 in Sorocaba-Votorantim and 19 in Rio Claro-Limeira, occurring mainly in conserved remnants of Atlantic Rain-forest, secondary growths, marshes and open fields. Whereas fireflies (Lampyridae) are found in almost all habitats, click beetles (Elateridae) and railroadworms (Phengodidae) occur predominantly in woody environments. Urban sprawl has an evident impact on the biodiversity of this selected group of beetles, and special attention should be given to the effect of artificial night lighting in the occurrence of these beetles. The lampyrids Aspisoma lineatum Gyllenhal, 1817 (Lampyrinae: Cratomorphini) and Bicellonychia lividipennis Motschulsky 1854 (Photurinae) are the most common species in urbanized areas, whereas many other species of lampyrids, elaterids and phengodids are rarely found in such environments. These studies offer insights on the potential use of a selected group of luminescent beetles found in this region as nocturnal environment bioindicators.

Keywords: Lampyridae, Elateridae, Phengodidae, fireflies, railroad-worms, click-beetles, bioluminescence, bioindicators, light pollution.
\end{abstract}

VIVIANI, V.R., ROCHA, M.Y. \& HAGEN, O. Fauna de besouros bioluminescentes (Coleoptera: Elateroidea: Lampyridae; Phengodidae, Elateridae) nos municípios de Campinas, Sorocaba-Votorantim e Rio Claro-Limeira (SP, Brasil): biodiversidade e influência da urbanização. Biota Neotrop. 10(2): http://www.biotaneotropica.org.br/v10n2/pt/abstract?article+bn03010022010.

Resumo: O Brasil abriga a maior biodiversidade de besouros bioluminescentes do mundo. Apesar disto, levantamentos faunísticos regionais e estudos sobre o efeito da urbanização na biodiversidade de vaga-lumes são inexistentes. Este tipo de estudo pode ser especialmente importante para selecionar novos bioindicadores de ambientes noturnos. A biodiversidade de espécies bioluminescentes de Elateroidea em três grandes áreas urbanas no Estado de São Paulo: os municípios de Campinas, Sorocaba-Votorantim e Rio Claro-Limeira foi catalogada. Em Campinas, a ocorrência de espécies tem sido monitorada durante os últimos 20 anos. Foi registrada a ocorrência de 26 espécies em Campinas, 21 em Sorocaba e 19 em Rio Claro, sendo estas encontradas principalmente em áreas de Mata Atlântica, crescimentos secundários, brejos e campos. Apesar de vaga-lumes lampirídeos serem encontrados em todos os habitats, elaterídeos e fengodídeos ocorreram encontrados preferencialmente em áreas florestadas. A expansão urbana, em especial a iluminação artificial tem impacto evidente na biodiversidade deste grupo de insetos. Os lampirídeos Aspisoma lineatum Gyllendal, 1817 (Lampyrinae: Cratomorphini) e Bicellonychia lividipennis Motschulsky, 1854 (Photurinae) são as espécies mais frequentes em areas urbanizadas, ao passo que várias outras espécies de lampirídeos, elaterídeos e fengodídeos são as mais incomuns nestas áreas. Estes estudos oferecem subsídios para o uso deste grupo de vaga-lumes como potenciais bioindicadores noturnos.

Palavras-chave: Lampyridae, Elateridae, Phengodidae, vaga-lumes, larvas-trenzinho, vaga-lumes tec-tec, bioluminescência, bioindicadores, poluição luminosa. 


\section{Introdução}

Vagalumes são coleópteros bioluminescentes pertencentes a três principais famílias da superfamília Elateroidea: Lampyridae (vaga-lumes), Phengodidae (trenzinhos) e Elateridae (vaga-lumes tec-tec). A bioluminescência nestas espécies é emitida por diferentes partes do corpo, na forma de diferentes padrões de sinalização e cores, para finalidades principalmente de reprodução e defesa (Lloyd 1983). Em vagalumes lampirídeos, machos e fêmeas se comunicam por padrões de sinalização característicos de cada espécie (Lloyd 1983). Elaterídeos e fengodídeos também podem utilizar a luminescência para reprodução embora os detalhes de sua comunicação ainda sejam pouco conhecidos (Bechara 1988, Lall et al. 2009), podendo utilizar a luminescência para fins de defesa.

Além da beleza aestética, os vagalumes tem adquirido importantes aplicações em diferentes campos, como em controle biológico de caramujos daninhos a agricultura (Bess 1956) e vetores de verminoses (Okada 1928, Viviani 1989a), e principalmente em biotecnologia como fonte de reagentes para análises de ATP e biomassa, e como marcadores bioluminescentes de expressão gênica (Viviani 2007).

O Brasil abriga a maior biodiversidade de espécies de coleópteros luminescentes do globo, com ca. 500 espécies descritas (Costa 2000), correspondendo a $\mathrm{ca}$ de $23 \%$ das espécies conhecidas no mundo. Revisões taxonômicas detalhadas em Elateridae luminescentes foram feitas para as tribos Pyrophorini e Campyloxenini (Agrypinae) (Costa 1975). Entretanto, as famílias Lampyridae e Phengodidae carecem de revisões mais recentes, e a identificação de espécies sul-americanas ainda permanece difícil. Estudos sobre a biologia e ecologia foram realizados para espécies de Elateridae (Costa 1975, Costa et al. 1988) e algumas espécies de Lampyridae e Phengodidae (Viviani \& Bechara 1997, Costa et al. 1999). Na família Lampyridae, estudos bionômicos foram feitos para espécies de Aspisoma spp Laporte 1833 (Costa 1988, Viviani 1989b). Viviani (2001) sumarizou aspectos biológicos de várias espécies de lampirídeos do Estado de São Paulo e Rosa (2007) descreveu os estágios imaturos e a bionomia de Photuris fulvipes Blanchard 1837 (Photurinae). Viviani (1989) publicou uma nota com um primeiro levantamento das espécies que ocorriam em algumas localidades do município de Campinas. Entretanto, ainda não existem levantamentos faunísticos regionais detalhados sobre as três famílias.

Estudos utilizando coleópteros como bioindicadores tem focalizado as famílias Scarabeidae, Carabidae (muitos tratando da subfamília Cicindelinae) e Staphylinidae que incluem muitas espécies de solo (Bohac 1999, Rainio \& Niemelã 2003, Marinoni \& Ganho 2006, Durães et al. 2005). Estes táxons tem sido utilizados como biondicadores ambientais quando uma espécie indica a degradação ou recuperação do meio ambiente; ecológicos quando uma espécie ou grupo de espécies indicam a qualidade de um determinado habitat, ou de biodiversidade quando uma espécie ou grupo servem como indicador da biodiversidade de uma forma geral. Entretanto ainda existem poucos estudos sobre a utilização de espécies de vagalumes como bioindicadores. Devido à diversidade de habitats em que ocorrem e a sensibilidade a fatores ambientais, especialmente à poluição luminosa (Lloyd 2006), espécies de vaga-lumes constituem potenciais bioindicadores para avaliação de impacto ambiental. Em prefeituras do Japão, a espécie Luciola cruciata Motschulsky, 1854 (Luciolinae: Luciolini) tem sido utilizada como bioindicador para avaliação da recuperação de cursos de água, pois sua larva é aquática e predadora de caramujos aquáticos, sendo muito sensível às condições da água. O desaparecimento de espécies de vaga-lumes tem sido notado em várias regiões do globo nas proximidades de locais urbanos (Viviani 2001, DeCock \& Matthysen 2004, Lloyd 2006). Em especial, devido à sensibilidade à poluição luminosa, os vaga-lumes constituem potenciais bioindicadores noturnos (Lloyd 2006). Entretanto ainda não existem estudos detalhados a respeito.

Apesar da rica biodiversidade e potencialidade de uso como bioindicadores, no Brasil ainda não existem levantamentos faunísticos locais e estudos relacionando a biodiversidade de espécies luminescentes com impacto ambiental e urbanização. Estudos deste tipo podem ser úteis para selecionar espécies de vaga-lumes como potenciais bioindicadores noturnos.

Neste trabalho é relatado um levantamento detalhado de espécies que ocorrem em diferentes habitats em cinco municípios localizados no Planalto Central do estado de São Paulo, anteriormente coberto predominantemente por Mata Atlântica Estacional, a saber: município de Campinas, no qual é feito um acompanhamento desde 1983; Rio Claro e Limeira, cujo levantamento iniciou em 2003, e Sorocaba e Votorantim cujos estudos iniciaram em 2006. Estes levantamentos abordam também a influência da urbanização na diversidade de espécies locais.

\section{Materiais e Métodos}

\section{Locais de coleta}

Foram realizadas coletas e observações em áreas representativas das bacias hidrográficas do Rio Atibaia-Piracicaba (Campinas, Rio Claro e Limeira), e do Rio Sorocaba (Sorocaba e Votorantim). As coletas foram realizadas principalmente no período de setembro-abril para adultos e outubro-abril para as larvas, com frequência mensal. No município de Campinas, foram feitas coletas e observações ao longo dos últimos 20 anos. Nos municípios de Rio Claro e Limeira, localizados a ca de $100 \mathrm{~km}$ de Campinas, os levantamentos começaram em 2003. No município de Sorocaba, localizado a $75 \mathrm{~km}$ de Campinas, as observações iniciaram em 2006. Estes municípios estão localizados na região morfoclimática de Mata Atlântica Estacional do Planalto Central Paulista, sendo que Rio Claro e Sorocaba também apresentam áreas de Cerrado, Floresta Estacional Semi-decídua, crescimentos secundários, campos e áreas palustres.

Descrição das áreas de coleta e observação. Foram investigados os seguintes habitats em diferentes localidades da região compreendendo os tres municípios: (I) Floresta Estacional Semidecídua; (II) crescimentos secundários; (III) mata de brejo; (IV) campos abertos e cerrados; (V) campos úmidos e depressões brejosas. A Figura 1 mostra alguns dos habitats mais representativos investigados. As áreas foram classificadas em 3 categorias principais de acordo com o estado de urbanização: (A) áreas bem preservadas consistindo de grandes áreas remanescentes de Floresta Atlântica estacional superiores a 1.000.000 $\mathrm{m}^{2}$ margeados por matas secundárias e àreas abertas em fase de sucessão; (B) áreas rurais sujeitas à ação antrópica através de atividades como agricultura e gado, dominadas por pastagens, áreas abandonadas, córregos não poluídos por esgoto doméstico, brejos e pequenos remanescentes de crescimentos secundários de floresta estacional com áreas entre $1.000-10.000 \mathrm{~m}^{2}$; (C) áreas semi-urbanas ou completamente urbanizadas com eventuais terrenos abandonados ou parques de tamanhos variáveis entre $300-50.000 \mathrm{~m}^{2}$, localizados entre residências

\section{Campinas $\left(22^{\circ} 44^{\prime} 45^{\prime \prime} S\right.$ e $\left.47^{\circ} 06^{\prime} 33^{\prime \prime} \mathrm{W}\right)$}

A Figura 2 mostra o mapa hidrográfico da região de Campinas investigada, compreendendo os bairros Vila Nogueira, Jardim das Paineiras e distrito de Sousas. mostrando os sítios onde foram efetuadas coletas.

Vila Nogueira (C). Coletas e observações neste bairro foram feitas entre 1981-1983. Neste período, o bairro já estava quase completamente urbanizado. Foram amostrados 4 terrenos baldios entre habitações de 300-1000 $\mathrm{m}^{2}$ numeradas de VN1 a VN4, 


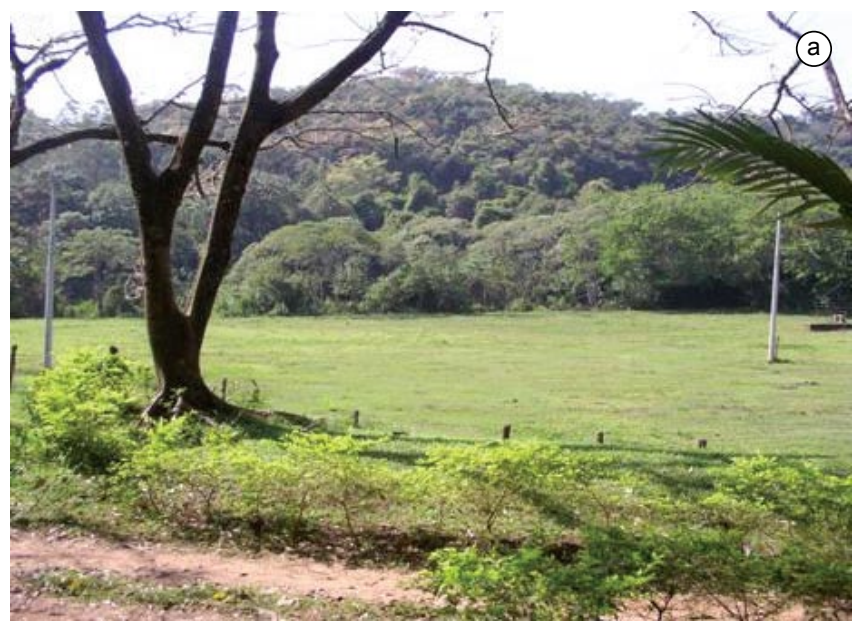

(C)

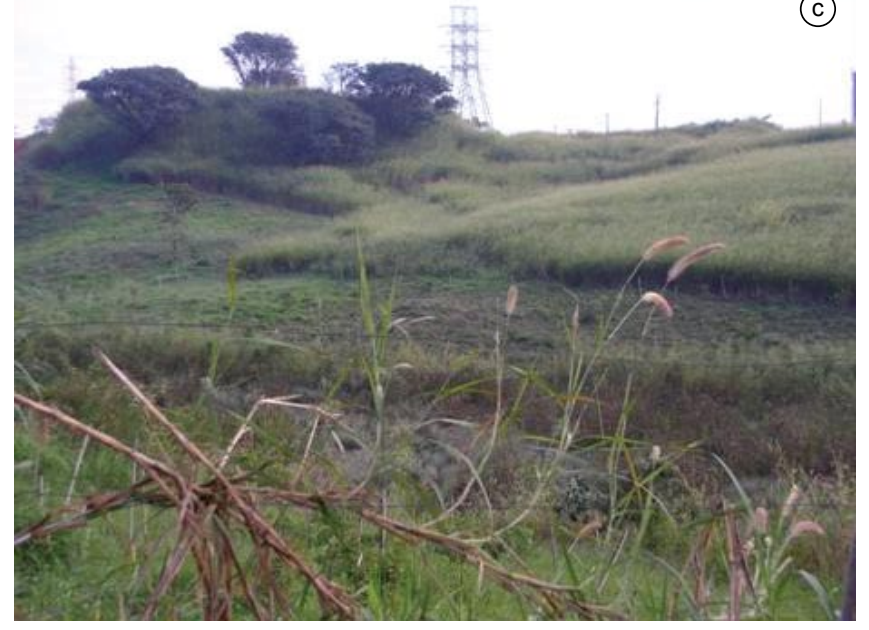

(b)
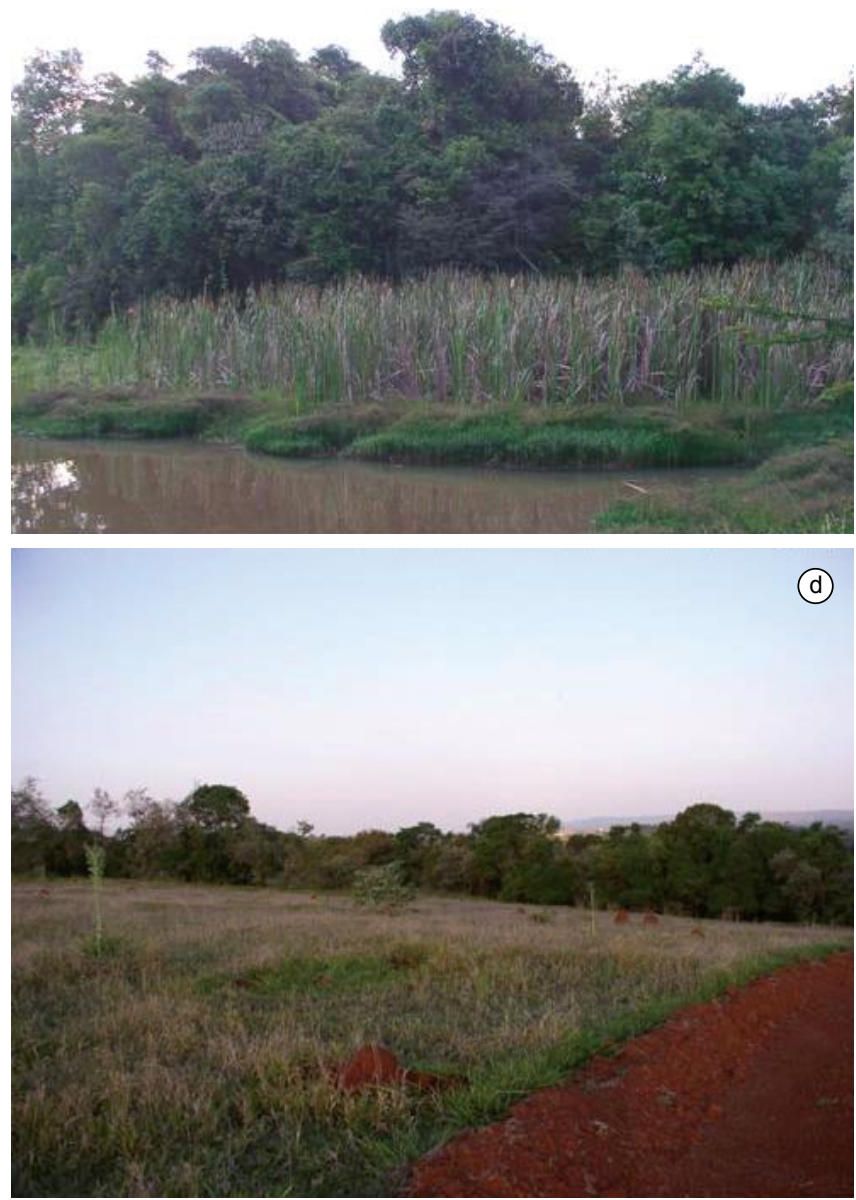

Figura 1. Habitats representativos das áreas deste estudo: a) Fazenda Santana (Sousas, Campinas, Brasil) mostrando no plano da frente um pasto palustre e no fundo um morro com floresta estacional; b) área de sucessão mostrando em sequência lagoa, vegetação aquática, campo de taboas (Typha angustifolia L.) (Typhaceae) e crescimento secundário no campus de Sorocaba da Universidade Federal de São Carlos (Sorocaba, Brasil); c) área de campo aberto no Jardim das Paineiras em Campinas; d) área de Cerrado em sucessão previamente destinado a pastagem com crescimento secundário de floresta estacional ao fundo, no campus de Sorocaba da Universidade Federal de São Carlos.

Figure 1. Representative habitats of the investigated areas: a) Santana Farm (Sousas, Campinas, Brazil) showing in the front view a marshy pasture and in the back view a remnant of seasonal rain forest; b) area showing the succession between lake, water grass, cattails (Typha angustifolia L.) (Typhaceae) fields and a secondary growth of seasonal rain forest; at campus de Sorocaba of Universidade Federal de São Carlos (Sorocaba, SP, Brazil); c) open fields in Jardim das Palmeiras, Campinas municipality; d) succession from a pasture to savanna (Cerrado) with secondary growth, at Sorocaba campus of Universidade Federal de São Carlos (Sorocaba, SP, Brazil).

cobertos por vegetação herbácea e campos de Ricinus communis $L$. (Euphorbiaceae) (mamonas), um parque com vegetação herbácea (VN5) com um córrego e área de brejo coberto por taboas (Typha angustipholia L.) (Typhaceae) (VN6), e 2 áreas de brejo numeradas de VN7 e VN8. Uma destas áreas (VN6), com ca. $1.000 \mathrm{~m}^{2}$ de brejo parcialmente drenado, com vegetação rasteira e trechos com taboas, ocorria dentro da escola Regina Coutinho Nogueira.

Jardim das Paineiras. Observações e coletas neste bairro iniciaram em 1983 e continuam até o momento atual. Em 1983 este bairro ainda consistia principalmente de área predominantemente rural do tipo B, com pastagens de gado bovino (Figura 1c) e equino, uma ampla rede de córregos afluentes do Riacho Mato Dentro, diversas áreas palustres e pequenos trechos de crescimentos secundários associados. Os sítios de coleta e observação foram denominados de JP1-JP4. JP1 consistia de terreno baldio com vegetação herbácea; JP2 consistia de depressão brejosa formada pelo afluente do Córrego Mato Dentro, com dois trechos remanescentes de crescimentos secundários ( ca 500 - 1.000 m²) e extensas áreas de campinas; JP3 consistia de amplo terreno palustre com vegetação herbácea e cinturões de $T$. angustitifolia, ao lado de uma rua e com algumas residências dispersas; JP4 consistia de depressão brejosa formada por um afluente do Córrego Mato Dentro, com área de crescimento secundário na encosta do córrego, pasto de gado bovino em outro lado e algumas residências. Atualmente este bairro está quase completamente urbanizado com residências e lotes de terrenos baldios de 300-1.000 m², e algumas áreas remanescentes de pastagens e crescimentos secundários flanqueando-o, sendo classificado como área urbana tipo C. Algumas áreas foram drenadas (JP2) ou aterradas (JP3, JP4), e o Riacho Mato Dentro encontra-se parcialmente canalizado em grande parte de seu percurso.

Fazenda Santana, Sousas (Figura 1a). Esta fazenda é cortada pelo Rio Atibaia. Em uma margem do rio encontra-se um grande remanescente de Mata Atlântica Semidecidual de ca $1.000 .000 \mathrm{~m}^{2}$, envolto por áreas de campo e crescimentos secundários em sucessão, 


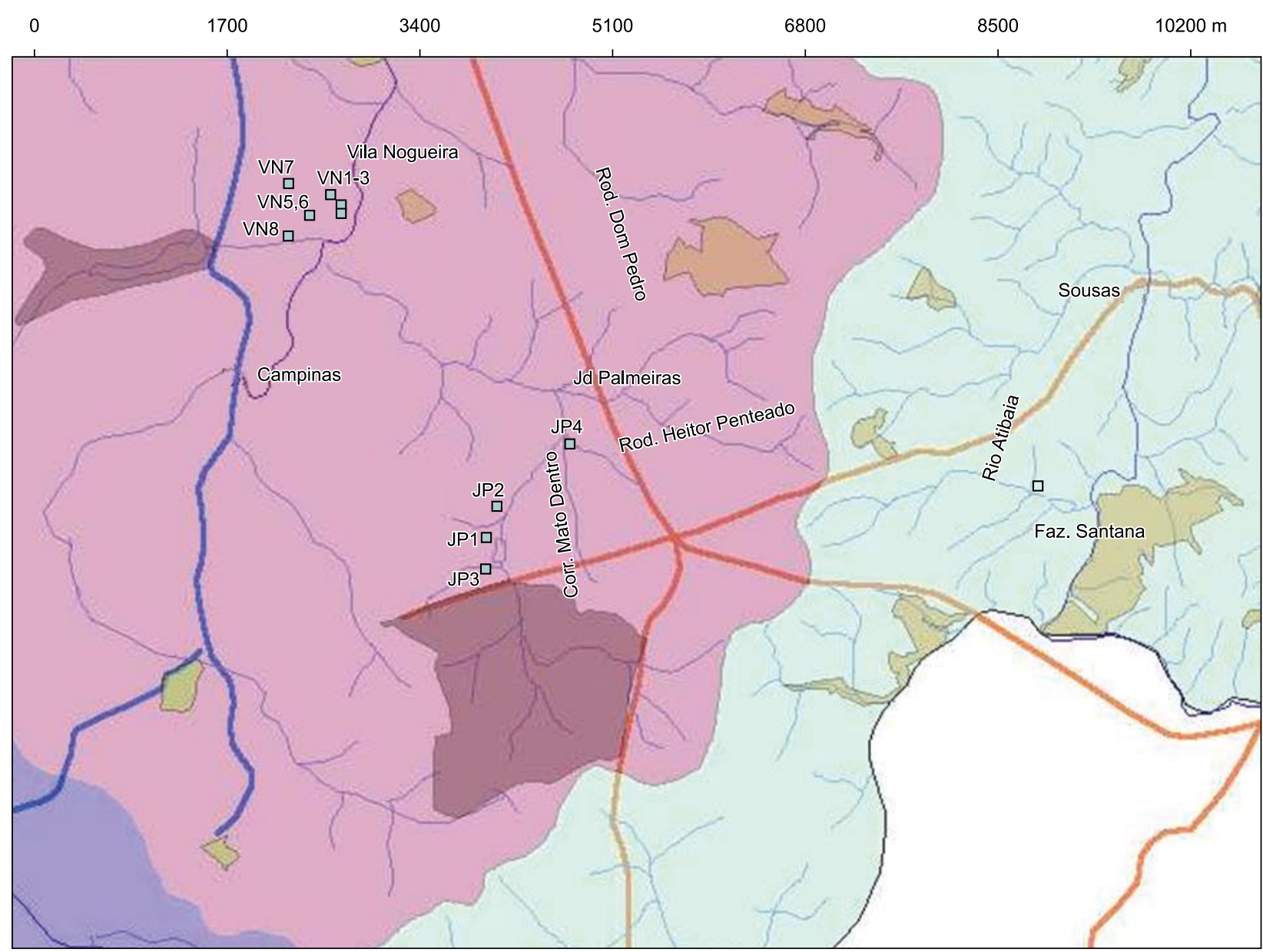

Figura 2. Mapa hidrográfico da região investigada em Campinas (Brasil) incluindo o Córrego Mato Dentro, o Rio Atibaia e os bairros Jardim das Paineiras, Vila Nogueira e distrito de Sousas. Os quadrados cheios representam os sítios investigados.

Figure 2. Hydrography of the investigated region in Campinas (Brazil), including the Mato Dentro Stream, Atibaia River and the neighborhood of Jardim das Palmeiras, Vila Nogueira and Sousas district. The filled squares represent the investigated sites.

área de Eucalyptus sp De Brutelle (Myrtaceae) e pastagens. Na outra margem, se encontra a área da fazenda com algumas moradias, pastos, pontos de crescimento secundários envolvendo vasta rede de córregos que formam brejos e lagos, sendo classificada como área preservada tipo A. Foram realizadas coletas dentro da mata, nas bordas da mata e nas àreas adjacentes, em brejos, e em pasto situado em local brejoso.

\section{Município de Sorocaba}

Bairro Alto da Boa Vista, Sorocaba (C). Área urbana localizada ao lado da Rodovia Senador Ermírio de Moraes altamente impactada, com terrenos baldios cobertos por vegetação herbácea baixa e área de crescimento secundário em torno de um córrego canalizado e iluminação artificial com lâmpadas de vapor de sódio.

Campolim (C). Área urbana que consiste de um parque de recreação de $c a .50 .000 \mathrm{~m}^{2}$ com áreas de gramado com árvores, área de campo aberto com mato rasteiro, brejo, dois córregos, um dos quais, parcialmente canalizado, e lago, no interior do bairro Campolim.

Campus da UFSCar, Bairro Itinga, Sorocaba ( $23^{\circ} 34^{\prime} 53$ ' $\mathrm{S}$ e 47 31' 28" W, $666 \mathrm{~m}$ ) (Figura 1b e 1d). Área rural em início de urbanização constituída por pastagens abandonadas, manchas de Cerrado, crescimentos secundários de floresta estacional, brejos, açudes e córregos.

\section{Município de Votorantim}

Bairro Jardim Clarice (B). Área urbana limítrofe com fazenda de gado em fase inicial de urbanização. Coletas foram realizadas em $\mathrm{JC} 1$ que consiste de depressão brejosa envolta por àrea de crescimento secundário impactada pelo gado, pastos e condomínio em fase de construção, e JC2 que consiste de área de crescimento secundário destinada a parque envolta por área residencial e pela fazenda de gado em outro lado.

\section{Município de Rio Claro}

UNESP Campus da Bela Vista (Rio Claro). Foram realizadas coletas e observações dentro do Campus em ampla área de campo aberto impactado, limítrofe com mata ciliar e campo de Eucalyptus sp do Horto Florestal

\section{Município de Limeira}

Charco Rodovia Washington Luís (Limeira) (Figura 1). Área constituída por pequeno açude $\left(c a 1.000 \mathrm{~m}^{2}\right)$ em estado de eutrofização, margeado por: a) área de brejo; b) pequena área de crescimento secundário interpondo-se entre o açude e vasta àrea de plantação de cana de açucar (Saccharum officinarum L.) (Poaceae); c) plantações de cana-de-açúcar e d) Rodovia Washington Luís. 
Mata de Brejo Rodovia Washington Luís (Limeira). Ampla área de mata de brejo, localizada próxima ao local anterior, margeada pela Rodovia Washington Luís por um alado, ampla área de campos úmidos, plantações de cana-de-açúcar e Rodovia Bandeirantes.

\section{Técnicas de coleta}

Lampirídeos. Adultos foram coletados no período de setembro a abril durante 1-2 horas após o por do sol com a ajuda de rede entomológica quando em vôo, ou manualmente quando pousados na vegetação.

Elaterídeos. Adultos foram coletados no período de outubrojaneiro. Foram localizados pela luminescência contínua em vôo ou pousados na vegetação e coletados com puçá, ou atraídos também com iscas de luz quimioluminescentes para pesca ou lanterna parcialmente encobertas.

Fengodídeos. Adultos do sexo masculino foram coletados com armadilha de luz ultravioleta e pano branco, e também, em raras ocasiões, no solo e na vegetação.

Larvas. Larvas de lampirídeos foram coletadas na vegetação, no solo ou na vegetação aquática à noite, mediante localização da luminescência relativamente contínua (Viviani 2001). Larvas de fengodídeos também foram coletadas à noite no solo das matas, por localização da luminescência (Viviani \& Bechara 1997). Larvas de elaterídeos foram raramente coletadas em troncos caídos ou no solo.

Identificação das espécies. Adultos e imaturos de vaga-lumes foram identificados por comparação com as seguintes coleções: (1) coleção de coleópteros bioluminescentes própria do autor na UFSCAR (Sorocaba, Brasil), (2) Museu de Zoologia da Universidade de São Paulo (MZUSP) e coleções do Museu de História Natural de Paris (França) e Museu de História Natural de Londres (Reino Unido). Os espécimes de elaterídeos foram identificados por C. Costa e Simone P. Rosa (MZUSP). Espécies de lampirídeos foram identificadas por V.Viviani e S. P. Rosa (MZUSP). Algumas espécies de lampirídeos e fengodídeos não puderam ser identificadas, e a estas foi atribuído um número específico, conforme Viviani \& Bechara (1997) e Viviani (2001). As seguintes morfoespécies foram recentemente identificadas por Simone P. Rosa (MZUSP) por comparação com espécimes do Museu de História Natural de Paris (França) : Cratomorphus sp. $4=$ C. gorhami $($ Lampyrinae: Cratomorphini); Pyrogaster sp.1- = Pyrogaster moestus (Photurinae); Amydetes sp.5 = Amydetes fanestratus (Lampyrinae: Amydetini). No campo, espécies de vaga-lumes lampirídeos em atividade foram identificadas também pelo característico padrão de sinalização bioluminescente de acordo com a Tabela 1. Os sinais bioluminescentes de várias espécies foram registrados também com vídeo câmera (Sony). Os espécimes foram depositados na coleção da UFSCar sob curadoria de V.Viviani e no MZUSP sob curadoria de C. Costa.

Medidas de intensidades de luz ambiental. Foram realizadas medidas de intensidades de luz ambiental em LUX com um fotômetro de campo ORIEL Goldilux e SKYE SKL310 em diferentes horários e habitats, para verificar o efeito da iluminação artificial na ocorrência de vaga-lumes.

\section{Resultados}

\section{Levantamento das espécies}

As Tabelas 2 e 3 sumarizam as espécies de lampirídeos, fengodídeos e elaterídeos encontradas em diferentes localidades e habitats nos cinco municípios. No total, 30 especies de coleópteros bioluminescentes da superfamília Elateroidea foram coletados nas famílias Lampyridae (22 spp.), Elateridae (5 spp.) e Phengodidae (3 spp.). Dentre estes, 26 espécies ocorreram em Campinas, sendo : 19 Lampyridae; 3 Phengodidae e 4 Elateridae. A região de Sorocaba e Votorantim apresentou 21 espécies, a maioria comum aos dois municípios. Os municípios de Rio Claro e Limeira contaram com 19 espécies. A ocorrência de algumas espécies tem variado ao longo dos anos em diferentes habitats de Campinas, mas ainda não temos estudos populacionais mais detalhados. Na família Lampyridae houve predominância da subfamília Lampyrinae (18 spp.), especialmente da tribo Cratomorphini (9 spp).

\section{Levantamento por habitat}

As Figuras 3 e 4 sumarizam algumas espécies mais representativas coletadas nos diferentes habitats amostrados. O maior número de espécies foi encontrado nas áreas mais preservadas de floresta estacional de Mata Atlântica, seguido de crescimentos secundários e seus arredores, brejos e campos (Tabela 3). Apesar disto, esta distribuição não foi uniforme dependendo da família (Figura 5).

Os lampirídeos foram encontrados em todos os habitats (Figura 5), inclusive em áreas impactadas como pastos e terrenos baldios ao lado de residências, enquanto que elaterídeos foram encontrados essencialmente em floresta estacional e crescimentos secundários. Os fengodídeos também foram encontrados predominantemente em áreas florestadas e, menos frequentemente, em campos.

Floresta Estacional Semi-decídua (Figura 1). No interior da mata, foram encontradas as espécies de lampirídeos Cratomorphus distinctus Olivier 1895, Cratomorphus sp.3, Photinus sp.1, Photinus sp. (Lampyrinae: Photinini) e Lucidota sp., os elaterídeos Pyrophorus divergens Eschscholtz, 1829 (Agrypinae: Pyrophorinae), Pyrearinus micatus Costa, 1978 (Pyrophorini) e Pyrearinus candelarius Germar, 1841e Hapsodrilus ignifer Germar 1824 (Pyrophorini) os fengodídeos Stenophrixothrix sp. e Phrixothrix hirtus e Pseudophengodes sp. (Phengodinae: Phengodini). Nas bordas da mata foram encontradas as espécies de lampirídeos Pyrogaster moestus Germar, 1824, Photinus sp.1, Aspisoma. physonotum Gorham, Cratomorphus concolor Perty, 1830, Cratomorphus gorhamii Olivier, os elaterídeos. Pyrophorus divergens, Pyrearinus micatus Costa, 1978 e Pyrearinus candelarius e o fengodídeo Stenophrixothrix.

\section{3. Áreas palustres}

Campos úmidos e depressões brejosas (Figura 1). Nestas áreas só foram encontradas espécies de lampirídeos. Em geral, as mesmas espécies foram encontradas em habitats similares nos municípios de Campinas, Sorocaba e Votorantim, e Rio Claro e Limeira. Foram encontradas Bicellonycha lividipennis Motschulsky, 1854 (Photurinae), B. ornaticollis Blanchard, 1837, Aspisoma sp.2 e Cratomorphus sp.2. As espécies B. lividipennis e B.ornaticollis são simpátricas e habitam campos úmidos, com solo parcialmente encharcado, sendo também encontradas frequentemente às margens de córregos e rios. As espécies Aspisoma sp.2 e Cratomorphus sp.2, foram encontrados em áreas mais alagadiças, frequentemente constituídas por vegetação herbácea parcialmente submersa e campos de taboas (Typha angustipholia) (Viviani 2001).

Mata ciliar e de brejo (Figura 1). Coletas e observações neste tipo de habitat foram feitas nas seguintes localidades: Horto Florestal (Rio Claro), Rodovia Washington Luís (Limeira). Neste habitat foram encontradas as espécies de lampirídeos Pyrogaster moestus, Photinus sp.1 e Aspisoma physonotum e no interior da floresta o elaterídeo Pyrophorus divergens.

Cerrado (Figura 1). Observações em áreas de cerrado foram realizadas no Campus da Bela Vista da UNESP (Rio Claro), e no campus da UFSCar de Sorocaba. Este habitat compartilha várias espécies de lampirídeos em comum com campos abertos como Aspisoma lineatum Gyllenhal, 1817, Aspisoma sp.4, Cratomorphus gorhami Olivier, C. concolor, Amydetes sp.1. Além destas espécies, foi encontrada Amydetes fanestratus Hoffmann unicamente na região 
Viviani, V.R. et al.

Tabela 1. Padrão de sinalização e características da bioluminescência das espécies (Coleoptera: Lampyridae, Phengodidae, Elateridae) investigadas.

Table 1. Signalling patterns and bioluminescence characteristics of the investigated species (Coleoptera: Lampyridae, Phengodidae, Elateridae).

\begin{tabular}{|c|c|c|c|}
\hline Taxon & Padrão & Pico Espectral (nm) & Cor \\
\hline \multicolumn{4}{|l|}{ LAMPYRIDAE } \\
\hline \multicolumn{4}{|l|}{ LAMPYRINAE } \\
\hline Cratomorphini & - & - & - \\
\hline Cratomorphus concolor Perty, 1830 & pulso/continuo & 554 & verde \\
\hline Cratomorphus distinctus Olivier, 1895 & pulso/continuo & 554 & verde \\
\hline Cratomorphus sp.2 & pulso/continuo & - & verde \\
\hline Cratomorphus sp. 3 & pulso/continuo & 551 & verde \\
\hline Cratomorphus gorhami Olivier, 1909 & pulso/continuo & 548 & Verde \\
\hline Aspisoma lineatum Gyllenhal, 1817 & flash bimodulado & 563 & verde-amarelo \\
\hline Aspisoma sp.4 & flash multimodulado & - & verde-amarelo \\
\hline Aspisoma physonotum Gorham, 1884 & pulso & 561 & verde-amarelo \\
\hline Aspisoma sp.2 & lento crescendo & 558 & verde-amarelo \\
\hline Photinini & - & - & - \\
\hline Photinus jenai & flash multimodulado & 567 & amarelo \\
\hline Photinus sp.1 & flash simples & 575 & amarelo \\
\hline Photinus sp. & flash simples & - & amarelo \\
\hline Macrolampis sp. & - & - & - \\
\hline Lucidotini & - & - & - \\
\hline Lucidota discoidalis Laporte, 1833 & continuo & 550 & verde \\
\hline Lucidota sp. & continuo & - & verde \\
\hline Incertae sedis & - & - & - \\
\hline Lamprocerini & - & - & - \\
\hline Lucio splendens Olivier, 1889 & - & - & - \\
\hline Amydetini & - & - & - \\
\hline Amydetes sp.1 & continuo & 548 & verde-azulado \\
\hline Amydetes fanestratus & continuo & 537 & verde-azulado \\
\hline \multicolumn{4}{|l|}{ PHOTURINAE } \\
\hline Photuris sp.7 & flash bimodulado & 552 & verde \\
\hline Bicellonychia lividipennis Motshulsky, 1854 & flash simples & 561 & verde-amarelo \\
\hline B. ornaticollis Blanchard, 1837 & flash simples & 562 & verde-amarelo \\
\hline Pyrogaster moestus Germar, 1824 & lento crescendo & 557 & verde-amarelo \\
\hline \multicolumn{4}{|l|}{ PHENGODIDAE } \\
\hline Mastinocerini & - & - & - \\
\hline Phrixotrix hirtus Olivier, 1909 & continuo & - & verde-amarelo \\
\hline Stenophrixotrix & continuo & - & amarelo \\
\hline Phengodini & - & - & - \\
\hline Pseudophengodes & - & - & - \\
\hline \multicolumn{4}{|l|}{ ELATERIDAE } \\
\hline \multicolumn{4}{|l|}{ AGRYPINAE } \\
\hline Pyrophorini & - & - & - \\
\hline Pyrophorus divergens Eschcholtz, 1829 & continuo & $545 / 568(\mathrm{P} / \mathrm{A})^{*}$ & verde/amarelo \\
\hline P. punctatissimus Blanchard, 1843 & continuo & $545 / 548(\mathrm{P} / \mathrm{A})^{*}$ & verde/verde \\
\hline Pyrearinus micatus Costa, 1978 & continuo & $540 / 540(\mathrm{P} / \mathrm{A})^{*}$ & verde/verde \\
\hline Pyrearinus candellarius Germar, 1841 & continuo & $544(\mathrm{P}) *$ & verde/verde \\
\hline Hapsodrilus ignifer Geramr, 1824 & continuo & $542 / 584(\mathrm{P} / \mathrm{A})^{*}$ & amarelo/amarelo \\
\hline
\end{tabular}

*(P/A): P: lanterna protorácica; A: lanterna abdominal 
Tabela 2. Espécies de coleópteros bioluminescentes (Coleoptera: Lampyridae, Phengodidae, Elateridae) encontradas em diferentes localidades nos municípios de Campinas, Sorocaba, Votorantim, Rio Claro e Limeira (Brasil).

Table 2. Bioluminescent Coleoptera species (Coleoptera: Lampyridae, Phengodidae, Elateridae) found in different localities in the municipalities of Campinas, Sorocaba, Votorantim, Rio Claro and Limeira (Brazil).

\begin{tabular}{|c|c|c|c|c|c|c|c|c|c|}
\hline \multirow[t]{2}{*}{ Taxon } & \multicolumn{3}{|c|}{ Campinas } & \multicolumn{3}{|c|}{ Sorocaba } & \multirow{2}{*}{$\begin{array}{c}\text { Votorantim } \\
\text { J. } \\
\text { Clarice } \\
\end{array}$} & \multirow{2}{*}{$\begin{array}{c}\begin{array}{c}\text { Rio } \\
\text { Claro }\end{array} \\
\text { UNESP }\end{array}$} & \multirow{2}{*}{$\begin{array}{c}\text { Limeira } \\
\text { R. W. } \\
\text { Luis } \\
\end{array}$} \\
\hline & $\begin{array}{c}\mathrm{V} . \\
\text { Nogueira } \\
\end{array}$ & $\begin{array}{c}\text { J. } \\
\text { Palmeiras } \\
\end{array}$ & $\begin{array}{c}\text { F. } \\
\text { Santana } \\
\end{array}$ & Campolim & $\begin{array}{l}\text { Boa } \\
\text { Vista }\end{array}$ & UFSCar & & & \\
\hline \multicolumn{10}{|l|}{ LAMPYRIDAE } \\
\hline \multicolumn{10}{|l|}{ LAMPYRINAE } \\
\hline Cratomorphini & - & - & - & - & - & - & - & - & - \\
\hline Cratomorphus concolor Perty, 1830 & $\mathrm{x}$ & $\mathrm{x}$ & $\mathrm{x}$ & - & $\mathrm{x}$ & $\mathrm{x}$ & $\mathrm{x}$ & $\mathrm{x}$ & - \\
\hline Cratomorphus distinctus Olivier, 1895 & - & - & $\mathrm{x}$ & - & - & $\mathrm{x}$ & - & - & - \\
\hline Cratomorphus sp. 2 & - & $\mathrm{x}$ & $\mathrm{x}$ & - & - & $\mathrm{x}$ & $\mathrm{x}$ & - & $\mathrm{x}$ \\
\hline Cratomorphus sp. 3 & - & - & $\mathrm{x}$ & - & - & - & - & - & - \\
\hline Cratomorphus gorhami Olivier, 1909 & - & - & $\mathrm{x}$ & - & - & $\mathrm{x}$ & $\mathrm{x}$ & $\mathrm{x}$ & - \\
\hline Aspisoma lineatum Gyllenhal, 1817 & $\mathrm{x}$ & $\mathrm{x}$ & $\mathrm{x}$ & $\mathrm{x}$ & $\mathrm{x}$ & $\mathrm{x}$ & $\mathrm{x}$ & $\mathrm{x}$ & $\mathrm{x}$ \\
\hline Aspisoma sp.4 & $\mathrm{x}$ & - & $\mathrm{x}$ & - & - & $\mathrm{x}$ & $\mathrm{x}$ & - & - \\
\hline Aspisoma physonotum Gorham, 1884 & - & - & $\mathrm{x}$ & - & - & $\mathrm{x}$ & - & - & $\mathrm{x}$ \\
\hline Aspisoma sp. 2 & $\mathrm{x}$ & $\mathrm{x}$ & $\mathrm{x}$ & - & - & $\mathrm{x}$ & $\mathrm{x}$ & - & $\mathrm{x}$ \\
\hline Photinini & - & - & - & - & - & - & - & - & - \\
\hline Photinus jenai & - & - & $\mathrm{x}$ & - & - & - & - & $\mathrm{x}$ & - \\
\hline Photinus sp.1 & - & $\mathrm{x}$ & $\mathrm{x}$ & - & - & $\mathrm{x}$ & $\mathrm{x}$ & $\mathrm{x}$ & - \\
\hline Photinus sp. & - & - & $\mathrm{x}$ & - & - & - & - & - & - \\
\hline Macrolampis sp. & - & - & - & - & - & - & $\mathrm{x}$ & - & - \\
\hline Lucidotini & - & - & - & - & - & $\mathrm{x}$ & - & - & - \\
\hline Lucidota discoidalis Laporte, 1833 & - & $\mathrm{x}$ & $\mathrm{x}$ & - & - & $\mathrm{x}$ & - & $\mathrm{x}$ & - \\
\hline Lucidota sp. & - & - & $\mathrm{x}$ & - & - & - & - & - & - \\
\hline Incertae sedis & - & - & $\mathrm{x}$ & - & - & - & - & - & - \\
\hline Lamprocerini & - & - & - & - & - & - & - & - & - \\
\hline Lucio splendens Olivier, 1889 & - & - & - & - & - & - & - & $\mathrm{x}$ & - \\
\hline Amydetini & - & - & - & - & - & - & - & - & - \\
\hline Amydetes sp.1 & $\mathrm{x}$ & $\mathrm{x}$ & $\mathrm{x}$ & $\mathrm{x}$ & $\mathrm{x}$ & $\mathrm{x}$ & $\mathrm{x}$ & $\mathrm{x}$ & - \\
\hline Amydetes fanestratus & - & - & - & - & $\mathrm{x}$ & $\mathrm{x}$ & $\mathrm{x}$ & - & - \\
\hline \multicolumn{10}{|l|}{ PHOTURINAE } \\
\hline Photuris sp.7 & - & - & - & - & - & - & $\mathrm{x}$ & - & - \\
\hline $\begin{array}{l}\text { Bicellonychia lividipennis Motshculsky, } \\
1854\end{array}$ & $\mathrm{x}$ & $\mathrm{x}$ & $\mathrm{x}$ & - & - & $\mathrm{x}$ & $\mathrm{x}$ & - & $\mathrm{x}$ \\
\hline B.ornaticollis Blanchard, 1837 & - & $\mathrm{x}$ & $\mathrm{x}$ & - & - & $\mathrm{x}$ & $\mathrm{x}$ & - & $\mathrm{x}$ \\
\hline Pyrogaster moestus Germar, 1824 & - & $\mathrm{x}$ & $\mathrm{x}$ & - & - & $\mathrm{x}$ & $\mathrm{x}$ & $\mathrm{x}$ & $\mathrm{x}$ \\
\hline \multicolumn{10}{|l|}{ PHENGODIDAE } \\
\hline Mastinocerini & - & - & - & - & - & - & - & - & - \\
\hline Phrixotrix hirtus Olivier, 1909 & - & $\mathrm{x}$ & $\mathrm{x}$ & - & - & - & - & - & - \\
\hline Stenophrixohtrix sp & - & - & $\mathrm{x}$ & - & - & $\mathrm{x}$ & - & $\mathrm{x}$ & - \\
\hline Phengodini & - & - & - & - & - & - & - & - & - \\
\hline Pseudophengodes sp & - & - & $\mathrm{x}$ & - & - & - & - & - & - \\
\hline \multicolumn{10}{|l|}{ ELATERIDAE } \\
\hline \multicolumn{10}{|l|}{ AGRYPINAE } \\
\hline Pyrophorini & - & - & - & - & - & - & - & - & - \\
\hline Pyrophorus divergens Eschchotz, 1829 & - & $\mathrm{x}$ & $\mathrm{x}$ & - & - & $\mathrm{x}$ & $\mathrm{x}$ & - & $\mathrm{x}$ \\
\hline P. punctatissimus Blanchard, 1843 & - & - & - & - & - & - & - & $\mathrm{x}$ & - \\
\hline Pyrearinus micatus Costa, 1978 & - & $\mathrm{x}$ & $\mathrm{x}$ & - & - & $\mathrm{x}$ & $\mathrm{x}$ & $\mathrm{x}$ & - \\
\hline Pyrearinus candellarius Germar, 1841 & - & $\mathrm{x}$ & $\mathrm{x}$ & - & - & $\mathrm{x}$ & $\mathrm{x}$ & $\mathrm{x}$ & $\mathrm{x}$ \\
\hline Hapsodrilus ignifer Germar, 1824 & - & - & $\mathrm{x}$ & - & - & - & - & - & - \\
\hline
\end{tabular}


Tabela 3. Ocorrência de espécies de besouros bioluminescentes (Coleoptera: Lampyridae, Phengodidae, Elateridae) em diferentes habitats nos municípios de Campinas, Sorocaba, Votorantim, Rio Claro e Limeira (Brasil).

Table 3. Occurrence of species of bioluminescent beetles in different habitats in the municipalities of Campinas, Sorocaba, Votorantim, Rio Claro e Limeira (Brazil).

\begin{tabular}{|c|c|c|c|c|c|c|c|}
\hline Taxon & $\begin{array}{c}\text { Campo } \\
\text { seco }\end{array}$ & $\begin{array}{l}\text { Cresc. } \\
\text { secund }\end{array}$ & $\begin{array}{c}\text { Floresta } \\
\text { estacional }\end{array}$ & $\begin{array}{c}\text { Mata brejo/ } \\
\text { ciliar }\end{array}$ & Brejo & Cerrado & Urbana \\
\hline \multicolumn{8}{|l|}{ LAMPYRIDAE } \\
\hline \multicolumn{8}{|l|}{ LAMPYRINAE } \\
\hline Cratomorphini & - & - & - & - & - & - & - \\
\hline Cratomorphus concolor & $\mathrm{x}$ & - & - & - & - & $\mathrm{x}$ & $\mathrm{x}$ \\
\hline Cratomorphus distinctus & - & - & $\mathrm{x}$ & - & - & - & - \\
\hline Cratomorphus sp.2 & - & - & - & - & $\mathrm{x}$ & - & - \\
\hline Cratomorphus sp.3 & - & - & $\mathrm{x}$ & - & - & - & - \\
\hline Cratomorphus gorhami & $\mathrm{x}$ & $\mathrm{x}$ & - & - & - & $\mathrm{x}$ & - \\
\hline Aspisoma lineatum & $\mathrm{x}$ & - & - & - & - & $\mathrm{x}$ & $\mathrm{x}$ \\
\hline Aspisoma sp4 & $\mathrm{x}$ & - & - & - & - & $\mathrm{x}$ & - \\
\hline Aspisoma physonotum & - & $\mathrm{x}$ & $\mathrm{x}$ & $\mathrm{x}$ & - & - & - \\
\hline Aspisoma sp.2 & - & - & - & - & $\mathrm{x}$ & - & - \\
\hline Photinini & - & - & - & - & - & - & - \\
\hline Photinus sp.1 & - & $\mathrm{x}$ & $\mathrm{x}$ & $\mathrm{x}$ & - & - & - \\
\hline Photinus sp. & - & - & $\mathrm{x}$ & - & - & - & - \\
\hline Macrolampis sp. & - & $\mathrm{x}$ & - & - & - & - & - \\
\hline Lucidotini & - & - & - & - & - & - & - \\
\hline Lucidota discoidalis & $\mathrm{x}$ & $\mathrm{x}$ & $\mathrm{x}$ & - & - & $\mathrm{x}$ & - \\
\hline Lucidota sp. & - & - & $\mathrm{x}$ & - & - & - & - \\
\hline Lamprocerini & - & - & - & - & - & - & - \\
\hline Lucio splendens & - & - & - & - & - & $\mathrm{x}$ & - \\
\hline Amydetini & - & - & - & - & - & - & - \\
\hline Amydetes sp.1 & $\mathrm{x}$ & $\mathrm{x}$ & - & - & - & $\mathrm{x}$ & $\mathrm{x}$ \\
\hline Amydetes fanestratus & $\mathrm{x}$ & $\mathrm{x}$ & - & - & - & $\mathrm{x}$ & - \\
\hline \multicolumn{8}{|l|}{ PHOTURINAE } \\
\hline Photuris sp7 & $\mathrm{x}$ & - & - & - & - & - & - \\
\hline Bicellonychia lividipennis & - & - & - & - & $\mathrm{x}$ & - & $\mathrm{x}$ \\
\hline B. ornaticollis & - & - & - & - & $\mathrm{x}$ & - & - \\
\hline Pyrogaster moestus & - & $\mathrm{x}$ & $\mathrm{x}$ & $\mathrm{x}$ & - & - & - \\
\hline \multicolumn{8}{|l|}{ PHENGODIDAE } \\
\hline Mastinocerini & - & - & - & - & - & - & - \\
\hline Phrixotrix hirtus & $\mathrm{x}$ & $\mathrm{x}$ & $\mathrm{x}$ & $\mathrm{x}$ & - & - & - \\
\hline Stenophrixotrix sp. & - & $\mathrm{x}$ & $\mathrm{x}$ & - & - & - & - \\
\hline Phengodini & - & - & - & - & - & - & - \\
\hline Pseudophengodes sp. & - & - & $\mathrm{x}$ & - & - & - & - \\
\hline \multicolumn{8}{|l|}{ ELATERIDAE } \\
\hline \multicolumn{8}{|l|}{ AGRYPINAE } \\
\hline Pyrophorini & - & - & - & - & - & - & - \\
\hline Pyrophorus divergens & - & $\mathrm{x}$ & $\mathrm{x}$ & $\mathrm{x}$ & - & - & - \\
\hline Pyrearinus micatus & - & $\mathrm{x}$ & $\mathrm{x}$ & $\mathrm{x}$ & - & - & - \\
\hline Pyrearinus candellarius & - & $\mathrm{x}$ & $\mathrm{x}$ & $\mathrm{x}$ & - & - & - \\
\hline Hapsodrilus ignifer & - & $\mathrm{x}$ & $\mathrm{x}$ & - & - & - & - \\
\hline
\end{tabular}



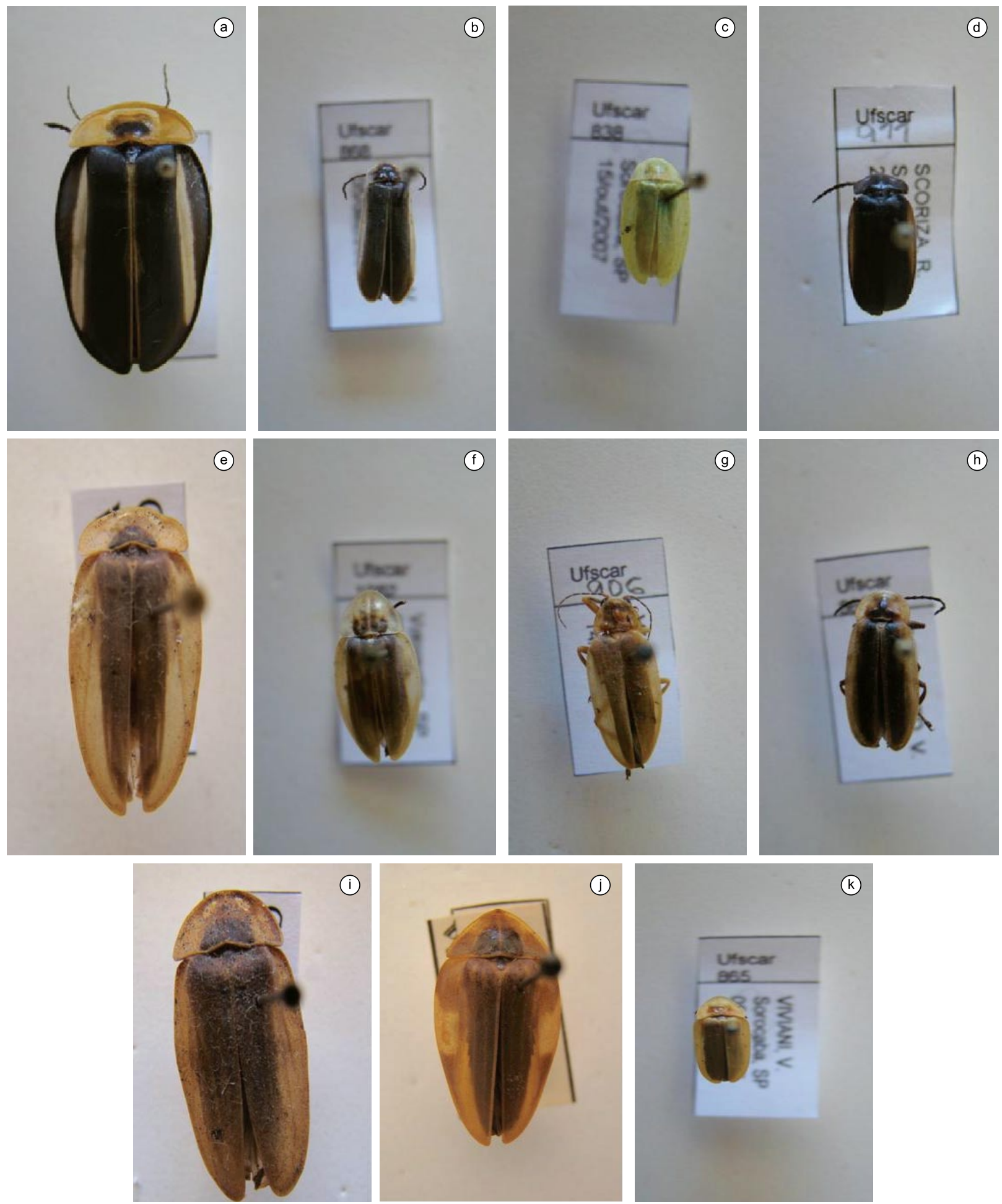

Figura 3. Algumas espécies de vaga-lumes lampirídeos (Coleoptera: Lampyridae) conspícuas em diferentes habitats na região de Campinas (Brasil): (Painel superior) espécies de floresta estacional e crescimentos secundários: a) Cratomorphus distinctus Olivier, 1895 (Lampyrinae: Cratomorphini); b) Photinus sp.1 (Lampyrinae: Photinini); c) Aspisoma physonotum Gorham 1884 (Lampyrinae: Cratomorphini) e d) Pyrogaster moestus Germar, 1824 (Photurinae); (Painel intemediário) espécies palustres: e) Cratomorphus sp.2 (Lampyrinae: Cratomorphini); f) Aspisoma sp.2 (Lampyrinae: Cratomorphini); g) Bicellonychia ornaticollis Blanchard, 1837 (Photurinae); h) Bicellonychia lividipennis (Photurinae); (Painel inferior) espécies campestres: i) Cratomorphus concolor Perty 1830 (Lampyrinae:Cratomorphini); j) Aspisoma lineatum Gyllhenal 1817 (Lampyrinae: Cratomorphini); k) Aspisoma sp.4 (Lampyrinae: Cratomorphini).

Figure 3. Some common firefly species (Coleoptera: Lampyridae) in different habitats of Campinas (Brazil): (Higher pannel) woody environment species found in seasonal rain forest and secondary growths: a) Cratomorphus distinctus Olivier, 1895 (Lampyrinae: Cratomorphini); b) Photinus sp.1 (Lampyrinae: Photinini); c) Aspisoma physonotum Gorham 1884 (Lampyrinae: Cratomorphini) e d) Pyrogaster moestus Germar, 1824 (Photurinae); (middle pannel) marshy species: e) Cratomorphus sp.2 (Lampyrinae: Cratomorphini); f) Aspisoma sp.2 (Lampyrinae: Cratomorphini); g) Bicellonychia ornaticollis Blanchard, 1837 (Photurinae); h) Bicellonychia lividipennis (Photurinae); (Lower pannel) open field species: i) Cratomorphus concolor Perty 1830 (Lampyrinae:Cratomorphini); j) Aspisoma lineatum Gyllhenal 1817 (Lampyrinae: Cratomorphini); k) Aspisoma sp.4 (Lampyrinae: Cratomorphini). 

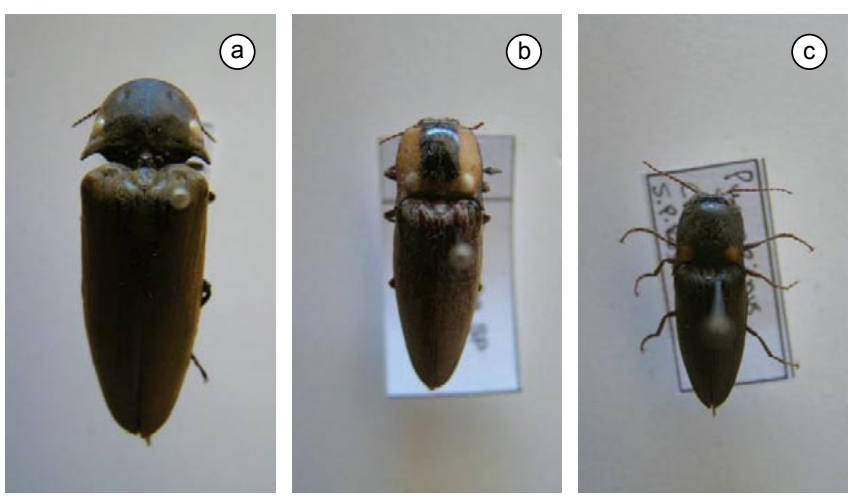

Figura 4. Elaterídeos (Coleoptera: Elateridae) luminescentes conspícuos em áreas de floresta estacional e crescimentos secundários: a) Pyrophorus divergens Eschscholtz, 1829 (Agrypinae: Pyrophorini); b) Pyrearinus candellarius Germar, 1841 (Agrypinae: Pyrophorini); e c) Pyrearinus micatus Costa, 1978 (Agrypinae: Pyrophorini).

Figure 4. Common luminescent click beetle species (Coleoptera: Elateridae) in areas of seasonal rain forest and secondary growths: a) Pyrophorus divergens Eschscholtz, 1829 (Agrypinae: Pyrophorini); b) Pyrearinus candellarius Germar, 1841 (Agrypinae: Pyrophorini); and c) Pyrearinus micatus Costa, 1978 (Agrypinae: Pyrophorini).

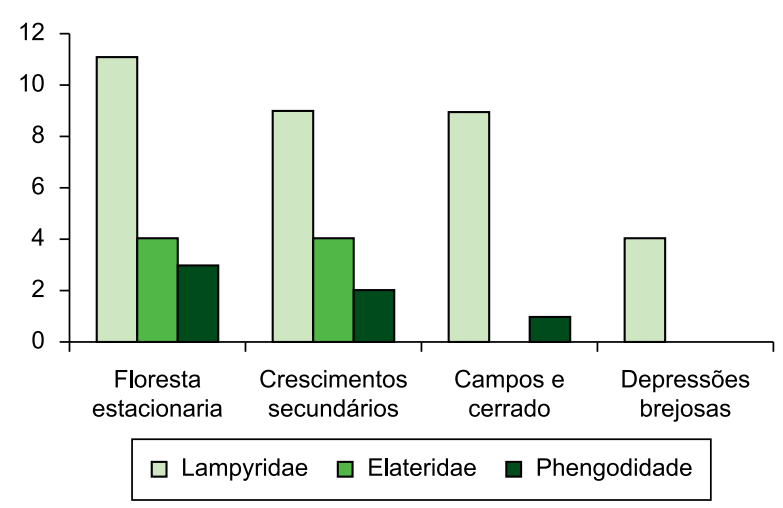

Figura 5. Número de espécies das familias de coleópteros bioluminescentes (Coleoptera: Lampyridae, Phengodidae, Elateridae) amostrados em diferentes habitats.

Figure 5. Number of species of bioluminescent Coleoptera families (Coleoptera: Lampyridae, Phengodidae, Elateridae) sampled in different habitats.

de Sorocaba. No campus da Bela Vista da UNESP (Rio Claro) foram encontrados os lampirídeos Photinus jenai, A. lineatum, C. concolor, C. gorhami, Amydetes sp.1, Lucio splendens Olivier, 1889, o fengodídeo Stenophrixothrix, e os elaterídeos Pyrophorus punctatissimus Blanchard, 1843 e P. candellarius, estas últimas duas espécies provavelmente vivem nas matas ciliares e em crescimentos secundários nos arredores.

Campos abertos (Figura 1). Observações em campo aberto foram feitas nos bairros Vila Nogueira, Jardim das Palmeiras em Campinas, Fazenda Santana (distrito de Sousas, Campinas), UNESP campus Bela Vista (Rio Claro) e Jardim Clarice (Votorantim). Na região de Campinas foram encontradas as espécies de lampirídeos A. lineatum, Aspisoma sp.4, C. concolor e Amydetes $\mathrm{sp}_{1}$ e eventualmente a espécie Phrixotrix hirtus. Na região de Sorocaba foram encontradas as espécies A. lineatum, Aspisoma sp.4, C. concolor e Amydetes sp.1, A. fanestratus, e em Rio Claro A. lineatum, Aspisoma sp.4, C. concolor, Amydetes sp.1 e P. jenai.

\section{4. Áreas impactadas e urbanizadas}

Pastos (Figura 1). Em pastos situados em áreas secas, frequentemente foram encontradas as espécies de lampirídeos A. lineatum, Aspisoma sp.4, C. concolor e C. gorhami, e eventualmente o fengodídeo Stenophrixothrix sp. No caso de pastos situados em locais brejosos, foram encontrados com mais frequência os lampirídeos B. lividipennis e A. lineatum.

Áreas urbanas. Foi analisada a ocorrência de espécies de vaga-lumes em áreas urbanas (Tabela 4; Figura 6). A Tabela 3 mostra a ocorrência geral de espécies nestas localidades, a Tabela 4 mostra a ocorrência de espécies em diferentes terrenos amostrados em 3 bairros, a Figura 6 resume o número de espécies em áreas com diferentes graus de urbanização e a Figura 7 mostra.a frequência com que as espécies mais comuns ocorrem no terrenos amostrados.

Vila Nogueira. Foram realizadas coletas num total de 8 terrenos abandonados (VN1-VN8), 5 constituídos por campos de vegetação preferencialmente herbácea ao lado de residências (VN1-VN5) e 3 constituídos por áreas de brejo (VN6-VN8). Em todos os terrenos baldios de vegetação herbácea (VN1-VN5) foi encontrada a espécie de lampirídeo A. lineatum. Em dois terrenos (VN1 e VN2), além desta espécie, encontramos também C. concolor, e em apenas um terreno (VN2) foi encontrada também a espécie Aspisoma sp.4.

$\mathrm{Na}$ área de campo úmido parcialmente drenado dentro da escola Regina Coutinho Nogueira (VN6 Bairro Vila Nogueira, Campinas), entre 1981-1983 havia B. lividipennis, e em seus arredores A. lineatum e Amydetes sp.1. Na mesma época, em depressão brejosa mais extensa em VN8, nas proximidades da escola, às margens do Córrego da Lagoa do Taquaral, foram encontradas populações de $B$. lividipennis e Aspisoma sp.2. Na parte brejosa da área VN5 não foi encontrada nenhuma espécie.

Jardim das Paineiras. Em terreno baldio próximo as residências (JP1) foi encontrada a espécie A. lineatum. No sítio JP2, constituído por depressão brejosa, flanqueada por pequenos trechos de crescimentos secundários e por campos foram encontradas 7 espécies de lampirídeos e 2 de elaterídeos (Tabela 4). No sítio JP4, semelhante ao JP2, foram encontradas 7 espécies de lampirídeos, 1 espécie de elaterídeo, e 1 espécie de fengodídeo.

No sítio JP3, que consistia de terreno pantanoso no Jardim das Palmeiras, onde haviam algumas habitações dispersas entre 1983-1984, foram observados e coletados espécimes de B. lividipennis. Em 1998 ainda foram observados sítios isolados com populações desta espécie na área. Entretanto, após loteamento e divisão por muros de cimento entre 2000-2002, restaram apenas alguns terrenos abandonados de natureza palustre, e esta espécie não foi mais encontrada no local.

Atualmente os sítios JP1, JP3 e JP4 foram aterrados e tomados quase completamente por residências. O sítio JP2 foi parcialmente aterrado e o córrego principal, Mato Dentro, foi drenado, restando pequena área de crescimento secundário e parte de brejo na margem de córrego afluente. Nesta última área atualmente encontramos unicamente a espécie $A$. lineatum, enquanto que as outras espécies não foram mais encontradas.

Jardim Clarice, Votorantim (B). Foram feitas coletas em dois sítios: JC1 consistindo de depressão brejosa contornada por pasto de gado bovino, trecho de crescimento secundário muito impactado pelo gado e por condomínio em fase de urbanização por outro lado; e JC2 que consiste de área de crescimento secundário destinada a parque, contornada por área residencial. Em JC1 encontramos 8 espécies de lampirídeos. Em JC2 foram encontradas 5 espécies de lampirídeos e 2 de elaterídeos (Tabela 4). 
Tabela 4. Ocorrência das espécies de coleópteros bioluminescentes (Coleoptera: Lampyridae, Phengodidae, Elateridae) mais comuns em diferentes sítios amostrados em áreas urbanas de Campinas e Sorocaba-Votorantim (Brasil).

Table 4. Occurrence of the most common bioluminescent Coleoptera species (Coleoptera: Lampyridae, Phengodidae, Elateridae) in different sites sampled in the municipalities of Campinas and Sorocaba-Votorantim (Brazil).

\begin{tabular}{|c|c|c|c|c|c|}
\hline $\begin{array}{l}\text { Vila Nogueira } \\
\text { (Campinas) }\end{array}$ & Espécies & $\begin{array}{c}\text { Jardim das } \\
\text { Paineiras (Campinas) }\end{array}$ & Espécies & $\begin{array}{l}\text { Jardim Clarice } \\
\text { (Votorantim) }\end{array}$ & Espécies \\
\hline $\begin{array}{c}\text { VN1 } \\
\text { (terreno baldio) }\end{array}$ & $\begin{array}{l}\text { A. lineatum, } \\
\text { C. concolor }\end{array}$ & JP1 (terreno baldio) & A. lineatum & $\begin{array}{c}\text { JC (depressão } \\
\text { brejosa, cresc. } \\
\text { Secundário) }\end{array}$ & $\begin{array}{c}\text { Aspisoma sp.2, } \\
\text { B. lividipennis, } \\
\text { B. ornaticollis, } \\
\text { Cratomorphus } \mathrm{sp} .2, \\
\text { A. lineatum, Aspisoma sp.4, } \\
\text { Amydetes fanestratus, } \\
\text { Pyrogaster moestus }\end{array}$ \\
\hline $\begin{array}{c}\text { VN2 } \\
\text { (terreno baldio) }\end{array}$ & $\begin{array}{l}\text { A. lineatum, } \\
\text { C. concolor, } \\
\text { Aspisoma sp. } 4\end{array}$ & $\begin{array}{c}\text { JP2 (depressão } \\
\text { brejosa, cresc. } \\
\text { Secundário) }\end{array}$ & $\begin{array}{c}\text { Aspisoma, } \\
\text { B. lividipennis, } \\
\text { B. ornaticollis, } \\
\text { Cratomorphus sp.2, } \\
\text { A. lineatum, } \\
\text { Photinus sp.1, } \\
\text { Pyrogaster moestus, } \\
\text { Pyrophorus divergens, } \\
\text { Pyrearinus micatus }\end{array}$ & $\begin{array}{c}\mathrm{JC} 2 \\
\text { (crescimento } \\
\text { secundário) }\end{array}$ & $\begin{array}{c}\text { Pyrogaster moestus, } \\
\text { Photinus sp.1, C. concolor, } \\
\text { A. lineatum, Amydetes } \\
\text { fanestratus, Pyreatinus } \\
\text { micatus, } P \text {. divergens }\end{array}$ \\
\hline $\begin{array}{c}\text { VN3 } \\
\text { (terreno baldio) }\end{array}$ & A. lineatum & JP3 (brejo) & $\begin{array}{l}\text { B. lividipennis, } \\
\text { A. lineatum }\end{array}$ & - & - \\
\hline $\begin{array}{c}\text { VN4 } \\
\text { (terreno baldio) }\end{array}$ & A. lineatum & $\begin{array}{c}\text { JP4 (depressão } \\
\text { brejosa, cresc. } \\
\text { secundário) }\end{array}$ & $\begin{array}{c}\text { Aspisoma sp. } 2 \text {, } \\
\text { B. lividipennis, } \\
\text { B. ornaticollis, } \\
\text { Cratomorphus } \mathrm{sp} .2 \text {, } \\
\text { C. concolor, } \\
\text { A. lineatum, Pyrogaster } \\
\text { moestus Pyrearinus } \\
\text { candellarius }\end{array}$ & - & - \\
\hline $\begin{array}{c}\text { VN5 } \\
\text { (terreno baldio) }\end{array}$ & A. lineatum & $\begin{array}{c}\text { JP5 (terreno brejoso } \\
\text { ao lado de residências) }\end{array}$ & $\begin{array}{l}\text { B. lividipennis, } \\
\text { Aspisoma sp. } 2\end{array}$ & - & - \\
\hline VN6 (brejo) & - & - & - & - & - \\
\hline VN7 (brejo) & $\begin{array}{l}\text { B. lividipennis, } \\
\text { A. lineatum }\end{array}$ & - & - & - & - \\
\hline VN8 (brejo) & $\begin{array}{l}\text { B. lividipennis, } \\
\text { Aspisoma sp. } 2\end{array}$ & - & - & - & - \\
\hline
\end{tabular}

\section{5. Áreas iluminadas}

Percebe-se que as áreas mais iluminadas são em geral as mais pobres em espécies luminescentes. Entre 10 sítios de campo aberto com iluminação de rua localizada a menos de $50 \mathrm{~m}$ de distância investigados nas áreas urbanas de Campinas e Sorocaba, 8 tinham a espécie $A$. lineatum, e dentre 7 sítios em locais brejosos, 5 apresentaram a espécie $B$. lividipennis. Nestas localidades, a iluminância ambiental, oriunda principalmente de lâmpadas de vapor de sódio, variava entre 0.2-0.85 LUX. A maioria das espécies investigadas até o momento ocorre em locais com luminosidade abaixo de 0.1 LUX.

\section{Sazonalidade}

Em virtude das observações feitas continuamente por um período superior a 20 anos na região de Campinas, por um período de 4 anos na região de Rio Claro, e 3 anos em Sorocaba, foi possível estabelecer a sazonalidade de um número considerável de espécies comuns a estas três regiões. A Tabela 5 resume o período anual de atividade observada para adultos de algumas espécies mais representativas na região. A maioria dos adultos das três famílias tem pico de atividade entre outubro e dezembro. Entre estas encontramos espécimes de lampirídeos $P$. moestus, A. physonotum, Aspisoma sp.4, C. concolor, C. distinctus, Cratomorphus sp.2, e a maioria dos elaterídeos e os fengodídeos. Algumas espécies de lampirídeos têm um espectro mais amplo de atividade, por exemplo, A. lineatum é encontrado em atividade no período de agosto ou setembro até abril. A espécie palustre Aspisoma sp.2 também apresenta dois picos anuais, um entre outubro-dezembro, e outro entre março-abril. Em anos recentes verificou-se que o período de atividade foi curiosamente estendido até os meses de fevereiro e março, para espécies como $P$. moestus, Photinus sp.1, que anteriormente tinham atividade até final de dezembro, e até março-abril, para espécies como B. lividipennis, cuja atividade era observada até final de fevereiro. 


\section{Discussão}

Neste trabalho mostramos a distribuição de espécies de coleópteros bioluminescentes da superfamília Elateroidea que ocorrem na região do Planalto Central do Estado de São Paulo, uma região relativamente urbanizada e desenvolvida em termos de agricultura. Uma grande parte

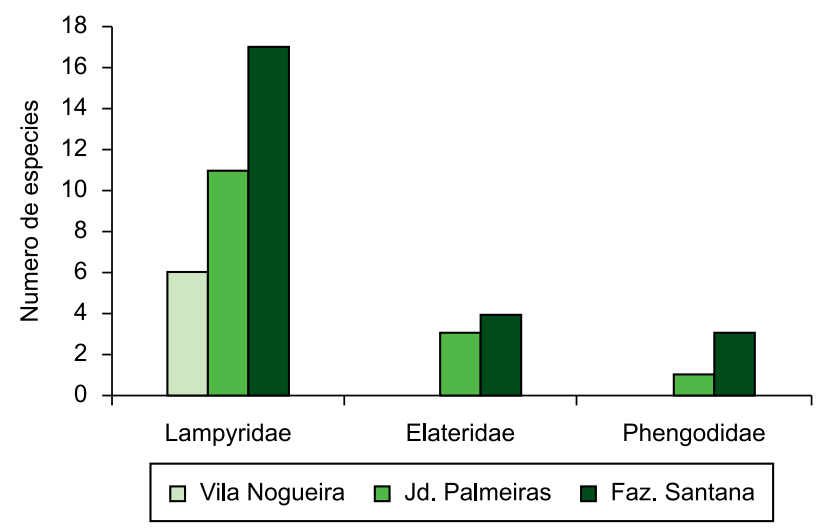

Figura 6. Número de espécies das famílias de coleópteros bioluminescentes (Coleoptera: Lampyridae, Phengodidae, Elateridae) amostradas em bairros em diferentes estágios de urbanização de Campinas (Brasil): a Fazenda Santana representa área tipo A, bem preservada; o bairro Jardim das Paineiras representava uma área tipo $\mathrm{B}$, predominantemente rural em início de urbanização, e o bairro Vila Nogueira constitui área tipo C completamente urbanizada.

Figure 6. Number of species of luminescent Coleoptera families (Coleoptera: Lampyridae, Phengodidae, Elateridae) sampled in neighborhoods under different stages of urbanization in Campinas municipality (Brazil): The Santana Farm represents a well preserved area type A; Jardim das Paineiras represented a rural area in the beginning of urbanization type B; and Vila Nogueira represents a totally urbanized area type C. das espécies estudadas e observadas é comum aos três municípios. É notável que as espécies Macrolampis sp., P. jenai e P. punctatissimus foram encontradas nas áreas de Cerrado em Sorocaba e Rio Claro também no Cerrado Central de Goiás (Com. pess.).

A floresta estacional apresentou o maior número de espécies, seguido de crescimentos secundários, campos e cerrado e brejos. Os lampirídeos foram encontrados em todos os habitats (Figura 5), enquanto que elaterídeos foram encontrados principalmente em floresta estacional e crescimentos secundários. Os fengodídeos foram encontrados predominantemente em áreas florestadas e menos frequentemente em campos (Figura 5). Estas diferenças refletem a ecologia destas espécies. Larvas de elaterídeos são carnívoras e vivem principalmente em troncos apodrecidos e no solo (Costa et al. 1988, Bechara 1988), o que pode justificar sua ocorrência em áreas florestadas mais ricas em nutrientes e biodiversidade. Fengodídeos se alimentam de diplópodes (Myriapoda: Diplopoda) (Viviani \& Bechara, 1997), encontrados principalmente no folhiço e em troncos em decomposição que ocorrem predominantemente em matas onde há abundância de madeira em decomposição, mas também em campos. Larvas de lampirídeos, por outro lado, são predadores de uma variada gama de espécies de gastrópodes (Mollusca: Gastropoda) (Viviani 2001), que podem ser de solo (lesmas), arbóreos como os caramujos Bradybaena semilaris Férussac, 1821 (Bradybaenidae: Bradybaeninae), Bulimulus tenuissimus d'Orbigni, 1835 (Bulimulidae: Bulimulinae) etc, ou aquáticos como os caramujos Lymnaea spp. (Lymnaeidae: Lymnaeninae) e Biomphalaria spp. (Planorbidae: Planorbinae), o que justificaria sua ocorrência em todos os habitats.

Como pode ser constatada, a urbanização tem profundo impacto na biodiversidade de espécies de coleópteros bioluminescentes (Figura 6). A maioria das espécies é afetada negativamente, desaparecendo dos locais urbanizados, enquanto que poucas espécies

Tabela 5. Sazonalidade dos adultos das principais espécies de besouros bioluminescentes (Coleoptera: Lampyridae, Phengodidae, Elateridae) estudadas.

Table 5. Sazonality of adults of the main species of bioluminescent beetles studied (Coleoptera: Lampyridae, Phengodidae, Elateridae).

\begin{tabular}{|c|c|c|c|c|c|c|c|c|c|c|c|c|}
\hline Taxon/ Mês & Ago. & Set. & Out. & Nov. & Dez. & Jan. & Fev. & Mar. & Abr. & Maio & Jun. & Jul. \\
\hline \multicolumn{13}{|l|}{ LAMPYRIDAE } \\
\hline \multicolumn{13}{|l|}{ Aspisoma lineatum } \\
\hline \multicolumn{13}{|l|}{ Aspisoma sp.2 } \\
\hline \multicolumn{13}{|l|}{ Aspisoma physonotum } \\
\hline \multicolumn{13}{|l|}{ Aspisoma sp.4 } \\
\hline \multicolumn{13}{|l|}{ Cratomorphus distinctus } \\
\hline \multicolumn{13}{|l|}{ C. concolor } \\
\hline \multicolumn{13}{|l|}{ Cratomorphus sp.2 } \\
\hline \multicolumn{13}{|l|}{ Cratomorphus sp.3 } \\
\hline \multicolumn{13}{|l|}{ Cratomorphus gorhami } \\
\hline \multicolumn{13}{|l|}{ Photinus sp.1 } \\
\hline \multicolumn{13}{|l|}{ Amydetes sp.1 } \\
\hline \multicolumn{13}{|l|}{ Amydetes fanestratus } \\
\hline \multicolumn{13}{|l|}{ Pyrogaster moestus } \\
\hline \multicolumn{13}{|l|}{ B. lividipennis } \\
\hline \multicolumn{13}{|l|}{ B. ornaticollis } \\
\hline \multicolumn{13}{|l|}{ ELATERIDAE } \\
\hline \multicolumn{13}{|l|}{ Pyrophorus divergens } \\
\hline \multicolumn{13}{|l|}{ Pyrearinus mictus } \\
\hline \multicolumn{13}{|l|}{ Pyrearinus candellarius } \\
\hline Hapsodrilus ignifer & & & & & & & & & & & & \\
\hline
\end{tabular}




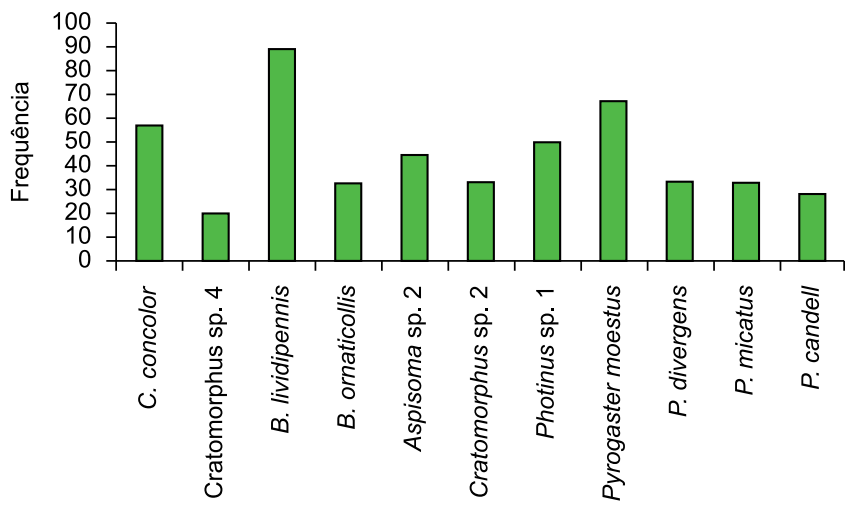

Figura 7. Frequência da ocorrência das espécies de coleópteros bioluminescentes (Coleoptera: Lampyridae, Phengodidae, Elateridae) mais comuns nos diferentes sítios amostrados em Campinas e Sorocaba-Votorantim (Brazil).

Figure 7. Frequency of the occurrence of the most common species of bioluminescent beetles in different sites sampled in Campinas and Sorocaba's municipalities.

podem eventualmente persistir e se adaptar. A Figura 7 mostra a frequência de ocorrência das espécies mais comuns nos respectivos habitats no total de sítios amostrados.

Espécies de floresta estacional são as mais sensíveis, ocorrendo unicamente em ambientes altamente sombreados e preservados. Espécies de lampirídeos de borda de matas como A. physonotum, P. moestus e Photinus sp.1, que são frequentemente encontrados também em crescimentos secundários, são dificilmente encontrados em fragmentos em áreas urbanas. Entre os elaterídeos, a espécie $P$. micatus é eventualmente encontrada em áreas urbanas, se áreas remanescentes de crescimentos secundários estiverem bem preservados.

Espécies de campo aberto são aparentemente menos afetadas. Em locais urbanos ainda podem ser encontradas espécies como A. lineatum, C. concolor e Aspisoma sp.4. Nos habitats mais impactados, como terrenos abandonados entre habitações a maioria das espécies desapareceu, entretanto poucas espécies foram persistentes ou até reincidentes. A espécie de lampirídeo A. lineatum é sem dúvida a espécie de campo aberto mais adaptável em locais urbanos. Amydetes fanestratus também tem sido encontrada em áreas bem impactadas proximas habitações na região de Sorocaba.

Em áreas palustres, B. lividipennis é a espécie mais frequente, enquanto que as espécies Cratomorphus sp. 2 e B. ornaticollis, não foram encontradas em áreas urbanas.

A análise dos fatores que influenciam esta diversidade é complexa. A influência antrópica pode se manifestar de diferentes formas: redução do habitat, criação de gado, agricultura, corte da vegetação, queimadas, lixo, e outros fatores como poluição do ar, sonora e luminosa, que são mais críticos próximos aos centros urbanos. Alguns fatores podem favorecer a persistência de certas espécies. A espécie $A$. lineatum pode ser encontrada próxima a hortas e jardins ricos em caramujos das espécies B. semilaris e B. tenuissimus. Em locais palustres próximos a pastagens, encontram-se frequentemente em abundância B. lividipennins e Aspisoma sp.2, Nestes locais é possível que os excrementos escoados pela chuva até os locais mais baixos favoreçam a proliferação de caramujos como Biomphalaria spp e Limnaea spp, e outros invetrebrados que sabidamente servem de alimento para estas espécies (Viviani, 2001).

Desta forma, os resultados sugerem que as espécies noturnas facilmente identificáveis pelo seu padrão de bioluminescência possam servir como bons indicadores. As espécies de lampirídeos
A. lineatum, B. lividipennis e Aspisoma sp.2 poderiam servir como indicadores de ambientes parcialmente impactados. As espécies de lampirídeos A. physonotum, P. moestus, Photinus sp.1, C. concolor e os elaterídeos $P$. micatus e $P$. candellarius podem servir como indicadores de floresta em fase de sucessão. Por outro lado, espécies como os lampirídeos C. distinctus, Cratomorphus sp.3, Photinus sp., os elaterídeos $P$. divergens e $H$. ignifer podem ser bons indicadores de floresta estacional em seu clímax.

Entre os fatores mais influentes, a iluminação artificial tem papel de destaque, pois é sabido que vagalumes dependem de sua bioluminescência para reprodução (Lloyd 1983), e sua atividade é afetada pelos níveis de iluminação ambiental (Lloyd 2006, Lall et al. 2009). Embora neste trabalho não tenham sido realizados estudos estatísticos mais detalhados, percebe-se claramente que locais mais iluminados são mais pobres em espécies bioluminescentes. Dos sítios amostrados, os mais ricos são aqueles que tem uma iluminância abaixo de 0.1 LUX, que é próximo ao valor de iluminância da lua cheia. Entretanto é notável que na maioria dos sítios amostrados próximos a iluminação de rua, com iluminância variando entre 0.2-0.85 LUX, ainda se encontram as espécies de lampirídeos A. lineatum e B. lividipennis. No caso de adultos de B. lividipennis, a atividade inicia após o crepúsculo, em intensidades de luz ambiental próximas de 4.5 LUX (Viviani 2001), portanto é esperado que estes indivíduos sejam menos afetados pela iluminação artificial abaixo deste valor. É possível que luz artificial de diferentes cromaticidades como aquela oriunda de lâmpadas de sódio ou mercúrio, afetem de forma diferente as espécies de vaga-lumes, dependendo do grau de sobreposição do espectro da fonte de luz com os espectros de bioluminescência e de sensibilidade óptica das espécies. Estudos estão em andamento para analisar e melhor entender o impacto da iluminação artificial na atividade e diversidade de vaga-lumes.

\section{Conclusões}

A biodiversidade de espécies luminescentes decresce consideravelmente com a urbanização, entretanto algumas espécies, especialmente A. lineatum, Amydetes sp.1 e B. lividipennis, frequentemente são persistentes ou até reincidentes. Outras espécies, como aquelas ativas na borda de matas e crescimentos secundários, são mais sensíveis e podem constituir potenciais bioindicadores noturnos. Os estudos sugerem que a iluminação artificial seja um dos principais fatores que afetam a ocorrência de vaga-lumes em seus habitats, afetando principalmente as espécies noturnas. Este estudo abre a possibilidade de se utilizar este grupo de besouros como potenciais bioindicadores noturnos de impacto ambiental em habitats de Floresta Atlântica Estacional, Cerrado e ambientes palustres na região.

\section{Agradecimentos}

Este estudo foi financiado pelo programa BIOTA da Fundação de Amparo à Pesquisa do Estado de São Paulo (FAPESP) e pelo Conselho Nacional de Desenvolvimento Científico e Tecnológico (CNPq) pela bolsa de produtividade (V. V). Agradecemos Cleide Costa (Museu de Zoologia da Universidade de São Paulo) pela leitura crítica do manuscrito e sugestões, e a Simone Policena Rosa (Museu de Zoologia da Universidade de São Paulo) pela identificação dos espécimes de lampirídeos, leitura e sugestões. Os autores agradecem também aos colegas que participaram das coletas ao longo destes anos, em especial Florisbela T. Ogawa (UNESP), Frederico Arnoldi (UNESP), Antonio Joaquim Silva Neto (UNESP), Priscila Tanioka (UFSCar), Valéria Scorsato (UFSCar) e Rogilene Prado (UFSCar). 


\section{Referências Bibliográficas}

BECHARA, E.J.H. 1988. Luminescent elaterid beetles: biochemical, biological and ecological aspects. In Advances in Oxygenated Processes (A.L. Baumastark, ed.) JAI Press London, p.123-178.

BOHAC, J. 1999. Staphylinid beetles as bioindicators. Agriculture, Ecossystems and Environment 74(1-3):357-372.

COSTA, C. 1975. Systematics and evolution of the tribes Pyrophorini and Heligmini with description of Campyloxeninae, a new subfamily. Arquiv. Zool. 26(2):49-190.

COSTA, C. 2000. Estado de Conocimiento de los Coleoptera Neotropicales. PRIBES (Martin-Pera, J.J.F., Morrone \& A. Melic, eds.). M3M Monografias Tercer Milenio, Zaragoza, p.99-114.

COSTA, C., VANIN, S.A. \& CESARI-CHEN, S.A. 1988. Larvas de Coleoptera do Brasil. Museu de Zoologia - Universidade de São Paulo, São Paulo.

COSTA, C., VANIN, S.A., CASARI, S.A. \& VIVIANI, V.R. 1999. Larvae of Neotropical Coleoptera. XXVII. Phrixothrix hirtus Olivier,1909, descriptions of immatures, neotenic female, adult male and and bionomic data (Phengodinae, Phengodidae, Coleoptera). Ilheringia. Ser. Zool. $86: 9-28$.

DE COCK, R. \& MATTHYSEN, E.2004. The adaptive value of luminescent behaviour in glow-worms (Coleoptera: Lampyridae). PhD Thesis, Universiteit Antwerpen, Antwerpen.

DURÃES, R., MARTINS, W.P. \& VAZ-DE-MELLO, F.Z. 2005. Dung beetles (Coleoptera: Scarabeidae): assemblages across a natural forest-Cerrado ecotone in Minas Gerais, Brazil. Neotropical Entomol. 34(5):721-731.

LALL, A., CRONIN, T.W., BECHARA, E.J.H., COSTA, C. \& VIVIANI, V.R. 2009. Visual ecology of bioluminescent beetles: visual spectral mechanisms and the colors of optical signaling in Coleoptera, Elateroidea: Lampyridae, Elateridae and Phengodidae. In Bioluminescence in focus-a collection of Illuminating essays: research signpost (V.B. Meyer-Rochow, ed.). Research Signpost, Kerala, p.201-228.

LLOYD, J.E. 1983. Bioluminescence and communication in insects. Annu. Rev. Entomol. 28:131-160.
LLOYD, J.E. 2006. Stray light, fireflies and fireflyers. In Ecological consequences of artificial night lighting. (C. Rich and T. Longcore, eds.). Island Press, Washington, DC, p.345-364.

MARINONI, R.C. \& GANHO, N.G. 2006. A diversidade diferencial beta de Coleoptera (Insecta) em uma paisagem antropizada do Bioma Araucaria. Rev. Bras. Entomol. 50(1):64-71.

OKADA, Y.K. 1928. Two aquatic glowworms. Trans. Ent. Soc. Lond. 1:101-107

RAINIO, J. \& NIEMELÃ, J. 2003. Ground beetles (Coleoptera: Carabidae) as bioindicators. Biodiversity and Conservation 12(3):487-506.

ROSA, S.P. 2007. Description of Photuris fulvipes (Blanchard) immatures (Coleoptera: Lampyridae, Photurinae) and bionomic aspects under laboratory conditions. Rev. Bras. Entomol. 51(2):125-130.

VIVIANI, V.R. \& BECHARA, E.J.H. 1997. Bioluminescence and biological aspects of Brazilian railroadworms (Coleoptera: Phengodidae). Ann. Entom. Soc Am. 90(3):389-398.

VIVIANI, V.R. 1989. Levantamento e ecologia das espécies de lampirídeos do município de Campinas. Bioikos 2(1):40-42.

VIVIANI, V.R. 1989a. A predação de caramujos Biomphalaria tenagophila por larvas de vagalumes lampirídeos. Bioikos 3(1):19-25.

VIVIANI, V.R. 1989b. Descricão dos estágios imaturos e dados biológicos de Aspisoma sp. (Coleoptera: Lampyridae). Rev. Bras. Entom. 33(2):359-366.

VIVIANI, V.R. 2001. Fireflies (Coleoptera: Lampyridae) from Southeastern Brazil: Habitats, Life History, and Bioluminescence. Ann. Entomol. Soc. Am. 94(1):129-145.

VIVIANI, V.R. 2007. Luciferases de vagalumes. Biotecnologia e Desenvolvimento 37:8-19.

Recebido em 23/12/09

Versão reformulada recebida em 14/05/10

Publicado em 08/06/10 